\title{
Micromachined Ultra Low-Power Sensor for Angular Resolved Flow Measurement
}

\author{
Samir Cerimovic ${ }^{1}$, Almir Talic ${ }^{2}$, Roman Beigelbeck ${ }^{2}$, Hannes Antlinger ${ }^{3}$, Franz Kohl ${ }^{2}$, Bernhard Jakoby ${ }^{3}$, \\ Franz Keplinger ${ }^{1}$ \\ ${ }^{1}$ Institute of Sensor and Actuator Systems, Vienna University of Technology, Vienna, Austria \\ ${ }^{2}$ Institute for Integrated Sensor Systems, Austrian Academy of Sciences, Wiener Neustadt, Austria \\ ${ }^{3}$ Institute for Microelectronics and Microsensors, Johannes Kepler University Linz, Linz, Austria
}

\section{Introduction and motivation}

Measurement of fluid velocity combined with an acquisition of flow direction is needed by many diverse applications such as environmental monitoring or industrial controlling systems. MEMS technology offers some decisive advantages that could not be achieved by conventional mechanical anemometers. Basically, the MEMS-based flow direction sensors can be divided into thermal and mechanical sensors, depending on their transduction method. The mechanical transducers utilize strain gauges mounted on the base of micromachined cantilevers, in order to measure the aerodynamic force acting on the beam [1]. These devices feature low power consumption but are prone to dirt due to the moving parts. On the other hand, miniaturized sensors based on the calorimetric principle consist typically of a resistive thinfilm heater surrounded by a conveniently arranged thermistor array, all embedded in a thin membrane [2]. They rely on flow dependent heat transfer altering the temperature distribution near the heater. These sensors offer high spatial and directional resolution at the expense of high power consumption (typically in the $\mathrm{mW}$ range) due to resistive heating. Consequently, the membrane over-temperature is relative high (usually in the range of 10 to $20 \mathrm{~K}$ [3]), which can be detrimental for some applications. In order to reduce the dissipated power, we developed a flow direction sensor based on a combined calorimetricanemometric transduction principle. The sensor utilizes the power efficient self-heating of the thermally coupled thermistors to induce the temperature field needed for flow detection. Due to extremely sensitive Ge-thermistors the power requirement is reduced significantly with respect to comparable calorimetric flow direction sensors $[4,5]$. According to FEM-simulations, the maximum difference between the membrane surface temperature and the ambient temperature is below $1 \mathrm{~K}$ even for gaseous fluids. The excess temperature of the fluid is so small that the related natural convection, which occurs if the sensor is tilted, causes only an imperceptible offset.

\section{Sensor design and fabrication}

The sensor is based on a circular array of eight Ge-Thermistors $\left(R_{\text {th1-8 }}\right.$, Fig. 1a). Two orthogonally arranged ensembles, each consisting of four thermistors, are connected to Wheatstone bridges supplied with a constant current (Fig. 1b). The bridge voltages offer information about the $x$ - and $y$-component of the flow velocity.

a)

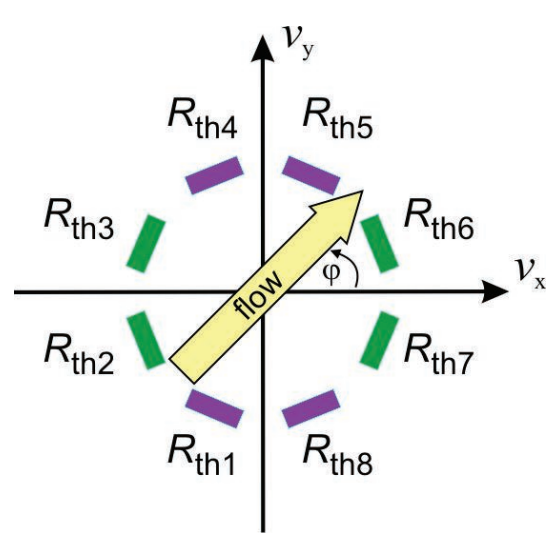

b)

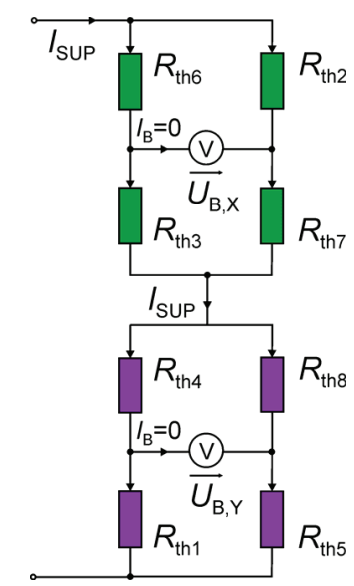

Fig. 1: a) Schematic layout of the sensor comprising eight Ge-thermistors $\left(R_{\text {th } 1-8}\right)$. The thermistors operate simultaneously as heat sources as well as temperature sensors. b) Two full bridge configurations are supplied with a constant electric current $I_{\text {SuP. }}$. 
Figure 2 shows a photomicrograph of the thermistor array, the thin micromachined membrane, and the supporting silicon frame. An additional thermistor placed on the silicon rim (outmost left in Fig. 2) enables the monitoring of the fluid temperature, which is in our measurement setup equal to the ambient temperature. The variation of the operating temperature does not affect the directional characteristics but only influences the flow velocity detection. Hence, it must be taken into account for precise measurement of all flow parameters.

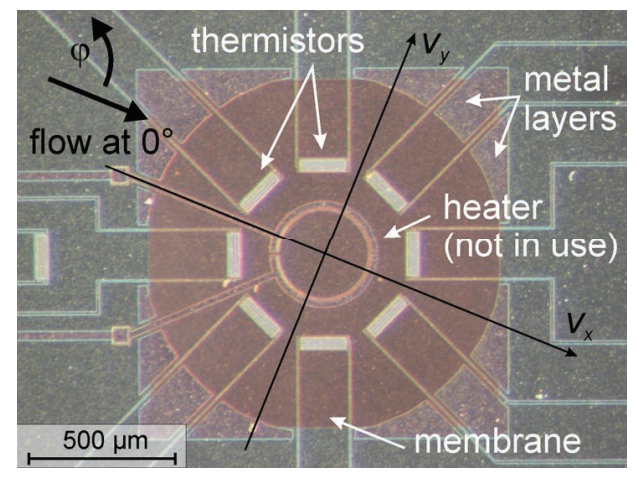

Fig. 2: Photomicrograph of the micromachined sensor. $\varphi$ denotes the flow direction. The bridge voltages depend on the $x$ - and $y$-component of the flow velocity. The axes indicate the directions of the $v_{x}$-and $v_{y}$-component of the velocity vector.

The sensor membrane is a sandwich structure consisting of the $\mathrm{SiO}_{2}$ and $\mathrm{Si}_{3} \mathrm{~N}_{4}$ wafer coating and the $\mathrm{SiN}_{\mathrm{x}}$ passivation layer, featuring a thickness of $250 \mathrm{~nm}, 70 \mathrm{~nm}$, and $1250 \mathrm{~nm}$, respectively. The central circular heater is not in use but could be utilized to operate the sensor in the standard calorimetric mode in order to compare the results. The only heat generating elements are the eight membrane thermistors placed symmetrically to membrane midpoint in a circular array with a $300 \mu \mathrm{m}$ radius. Each of them consists of a $260 \mathrm{~nm}$ thick germanium film, which is contacted by four metal strips (Ti-Au-Cr sandwich) exhibiting a total thickness of about $270 \mathrm{~nm}$. A single thermistor measures $400 \times 35 \mu \mathrm{m}^{2}$ and its resistance at a room temperature is typically $300 \mathrm{k} \Omega$. Amorphous germanium thermistors feature a negative temperature coefficient of resistivity (TCR) of about $-2 \% / \mathrm{K}$ enabling highly sensitive temperature measurmenets [6]. The sensor membrane has an overall size of about $1.2 \times 1.2 \mathrm{~mm}^{2}$ and is suspended over a $350 \mu \mathrm{m}$ thick micromachined silicon frame. The membrane was fabricated by a $\mathrm{KOH}-$ based anisotropic etching process from the backside of the wafer. This way of producing a membrane is rather simple, although the shape is restricted to rectangular forms. Consequently, some thermistors are lying closer to the silicon rim which influences their thermal behaviour. To reduce deviations from the rotational symmetry of the thermal system, metal layers are arranged at the corners, circularly surrounding the remaining membrane structure (Fig. 2). For more details regarding sensor fabrication technology refer to [7].

\section{FEM Simulations}

In order to study sensor behavior and to estimate the transduction characteristics as well as the overtemperature distribution, a 3D-FEM (Finite Element Method) model of the sensor was developed. Figure 3 shows the simulation results for the membrane area at zero flow $(v=0 \mathrm{~m} / \mathrm{s})$ and at a constant air flow velocity of $v=2 \mathrm{~m} / \mathrm{s}$ in $v_{x}$-direction $\left(\varphi=0^{\circ}\right)$.

For the thermistor arrangement as depicted in Fig. 1 the bridge voltages equals to

$U_{\mathrm{B}, \mathrm{X}}=I_{\mathrm{SUP}} \frac{R_{\mathrm{th} 2} R_{\mathrm{th} 3}-R_{\mathrm{th} 6} R_{\mathrm{th} 7}}{R_{\mathrm{th} 2}+R_{\mathrm{th} 3}+R_{\mathrm{th} 6}+R_{\mathrm{th} 7}}, \quad U_{\mathrm{B}, \mathrm{Y}}=I_{\mathrm{SUP}} \frac{R_{\mathrm{th} 1} R_{\mathrm{th} 8}-R_{\mathrm{th} 4} R_{\mathrm{th} 5}}{R_{\mathrm{th} 1}+R_{\mathrm{th} 4}+R_{\mathrm{th} 5}+R_{\mathrm{th} 8}}$.

At zero flow, the temperature field generated by the thermistors is approximately 8-fold rotational symmetric with respect to the membrane midpoint (Fig. 3a). Hence all thermistors measure the same temperature and consequently the bridges are in balance (i.e., $U_{B, X}=U_{B, Y}=0$ ). The convective heat transfer induced by the media flowing across the sensor surface disturbs the thermal symmetry. In the case of a flow in $v_{x}$-direction $\left(\varphi=0^{\circ}\right.$, Fig. $\left.3 \mathrm{~b}\right)$, the upstream thermistor pair of the $x$-bridge $\left(R_{\text {th2 }}\right.$ and $\left.R_{\text {th } 3}\right)$ is cooled down more intensively than its downstream counterpart $\left(R_{\mathrm{th} 6}\right.$ and $\left.R_{\mathrm{th} 7}\right)$. Due to the negative TCR of the thermistors, the difference in the numerator of left side in equation (1) increases with increasing 
a)

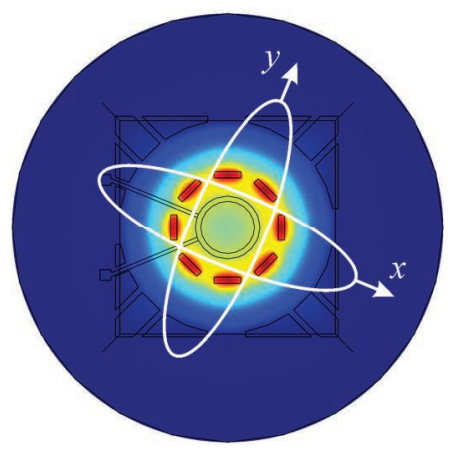

b)

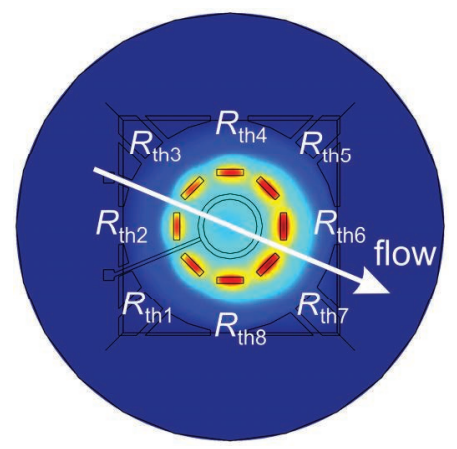

Fig. 3: a) Simulation results for the membrane area at zero flow. The two orthogonal thermistor ensembles and the corresponding axes are indicated. As the temperature field is rotational symmetric, all thermistors measure the same temperature. b) At $v=2 \mathrm{~m} / \mathrm{s}$ the convective heat transfer disturbs the thermal symmetry which influences the bridge output signals (flow is in $v_{x}$-direction, $\varphi=0^{\circ}$ ).

flow velocity. On the other hand, the upstream thermistors of the $y$-bridge $\left(R_{\text {th } 1}\right.$ and $\left.R_{\text {th } 4}\right)$ and the downstream ones $\left(R_{\text {th5 }}\right.$ and $\left.R_{\text {th8 }}\right)$ measure the same temperature, respectively. Therefore, the $U_{\mathrm{B}, Y}$ equals zero for $\varphi=0^{\circ}$. Owing to the rotational symmetry as well as the orthogonal arrangement of the thermistor bridges, the directional characteristics of these two outputs are approximately sinusoidal with a 90 degree phase shift between each other (Fig. 4a). Therefore, the angle of the flow direction with respect to the positive $x$-direction can be calculated by

$\varphi=\arctan \left(\frac{U_{\mathrm{B}, \mathrm{Y}}}{U_{\mathrm{B}, \mathrm{X}}}\right)$

whereas the flow velocity is a function of the modulus of the bridge voltages

$\left|U_{\mathrm{B}}\right|=\sqrt{U_{\mathrm{B}, \mathrm{X}}^{2}+U_{\mathrm{B}, \mathrm{Y}}^{2}}$.

The function $\left|U_{B}\right|=f(v)$ must be obtained experimentally.

The maximum difference between the membrane surface temperature and the temperature of the silicon rim occurs when no convective cooling takes place. Due to supply current in the $\mu \mathrm{A}$ range, the membrane over-temperature is extremly low. At zero flow and nitrogen gas as fluid, it does not exceed the $0.5 \mathrm{~K}$ mark (Fig. 4b). Hence, the heating of the passing fluid can be neglected for almost all practical application.

a)

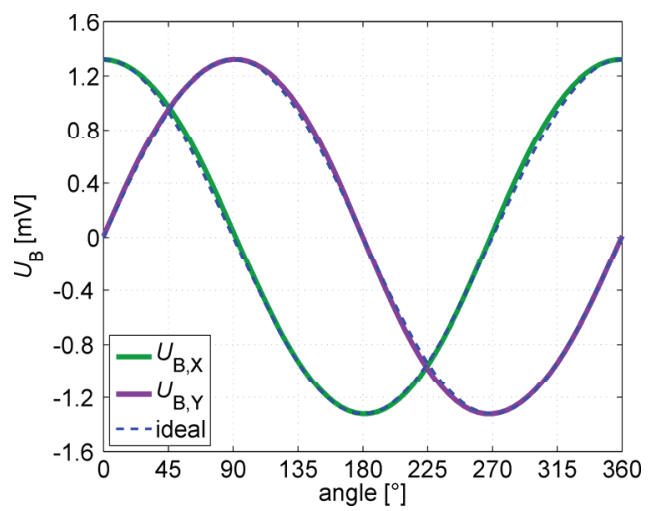

b)

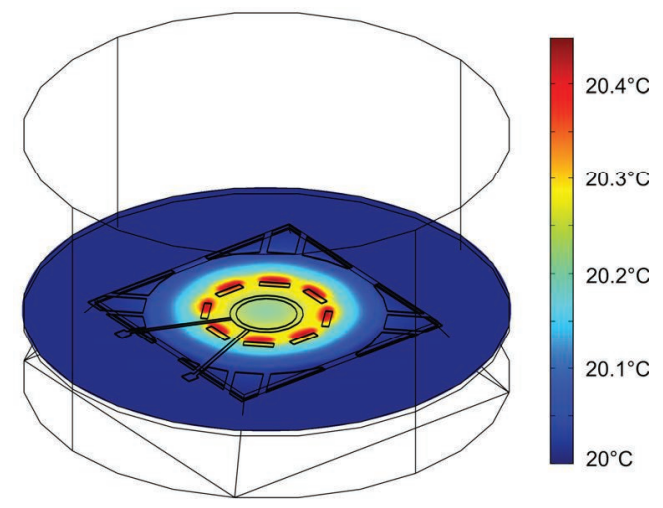

Fig. 4: a) Simulated directional characteristics of the two bridge voltages at $v=2 \mathrm{~m} / \mathrm{s}$. Due to symmetric circular arrangement the outputs feature approximately sinusoidal characteristic. The dashed lines represents ideal sine- and cosine-functions. b) 3D FEM simulation at zero flow $(v=0 \mathrm{~m} / \mathrm{s})$. The colour scale indicates that the maximum overtemperature is less than $0.5 \mathrm{~K}$ (supply current $\left.I_{\mathrm{SuP}}=8 \mu \mathrm{A}\right)$.

\section{Measurements and results}

The device characterization was performed with filtered nitrogen gas as a test fluid at a constant flow velocity of $2 \mathrm{~m} / \mathrm{s}$, averaged over the cross section of the channel. In order to establish reproducible flow directions the sensor chip was flush-mounted with surface of a fixed aluminium disk. The disk forms the 


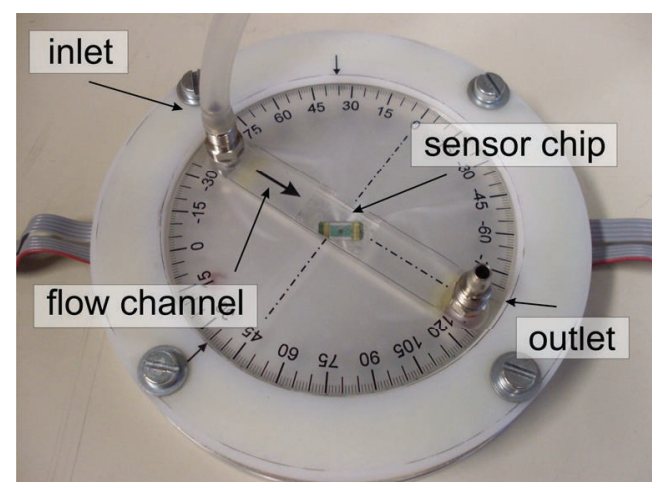

Fig. 5: A rotatable rectangular flow channel $\left(12 \times 1 \mathrm{~mm}^{2}\right)$ is placed above the sensor chip. The direction of the tangential nitrogen flow can be adjusted over the whole $360^{\circ}$ range.

bottom of a rotatable rectangular flow channel of $12 \mathrm{~mm}$ width and $1 \mathrm{~mm}$ height (Fig. 5). This setup allows $360^{\circ}$ rotation of the flow channel with an accuracy of $\pm 1^{\circ}$.

The double bridge was supplied with a DC current of only $8 \mu \mathrm{A}$ resulting in a dissipated power of less then $5 \mu \mathrm{W}$ per thermistor. Due to very low self-heating of the thermistors, the measured amplitudes of the bridge unbalance voltage are in the range of few $\mathrm{mV}$. In order to improve the signal-to-noise ratio lowpass filtering and data averaging must be applied, which diminishes the dynamic of the system.

Figure 6 illustrates the measured bridge voltages $U_{B, X}$ and $U_{B, Y}$ as a function of the flow direction. For both curves an offset in a range of few $\mathrm{mV}$ can be observed. Moreover, Fig. 6 reveals a slight difference in the peak-to-peak voltage of the bridge outputs. Both effects stem from the asymmetry in the membrane arrangement. It is not possible to fabricate completely identical thermistors and to ensure a perfect rotational symmetry of the thermal system. Hence, the bridges are unbalanced even at zero flow resulting in a constant offset of the output signal. One reason for the asymmetry is a shift of the membrane structure with respect to the rectangular silicon rim. Due to the mask misalignment generated by the backside lithography step prior to anisotropic etching process, some thermistors are shifted closer to the silicon bulk than others. This influences the temperature distribution and causes the mentioned amplitude deviations. Furthermore, any asymmetry caused by sensor mounting or measurement setup also affects the output characteristic.

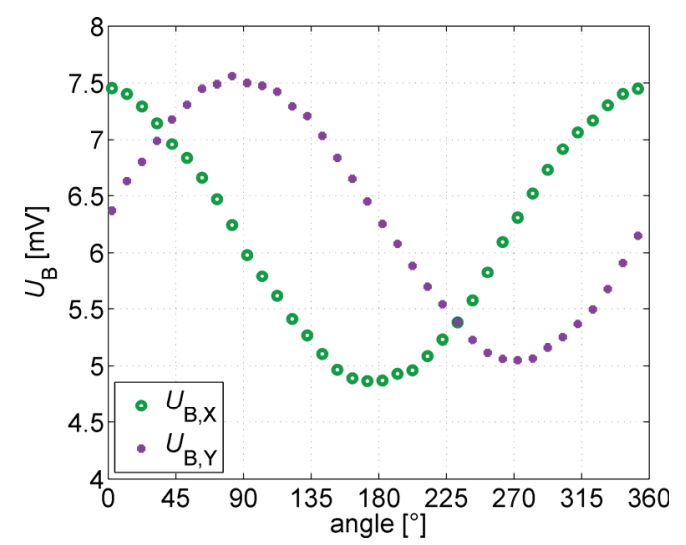

Fig. 6: Measured bridge voltages $U_{B, X}$ and $U_{B, Y}$ as a function of the flow direction $\varphi$ at $v=2 \mathrm{~m} / \mathrm{s}$ flow velocity. The signals contain initial offsets which are still to be removed.

As both the offset as well as the deviation of signal maxima are systematic errors, they can be taken into account by means of subsequent data processing. A functional block diagram of a rather simple but effective correction procedure is depicted in Fig. 7a. The bridge outputs are first low-pass filtered and then sampled by means of a DAC. The values for the offset correction $U_{\text {OFFSET,X-Y }}$ can be obtained by measuring the bridge voltages at zero flow. After the offset subtraction, the signal is multiplied with an appropriate correction factor depending on the sign of the offset-free bridge voltage. In order to calculate these correction factors the magnitude of $U_{X}$ at $\varphi=0^{\circ}$ was chosen as a reference amplitude. The magnitudes of the bridge voltages at $\varphi=90^{\circ}, 180^{\circ}$, and $270^{\circ}$ can be fitted to this reference by multiplying them with a constant value (for example $U_{X}=k_{90^{\circ}} \cdot U_{Y}$ for $U_{Y}$ at $\varphi=90^{\circ}$ ). Note that the correction factor $k_{0^{\circ}}$ must be 1 since the voltage $U_{X}$ at $\varphi=0^{\circ}$ was chosen as a reference. Figure $7 \mathrm{~b}$ shows the compensated output signals $X$ and $Y$ as a function of the flow direction at constant flow velocity. To illustrate the impact 
a)

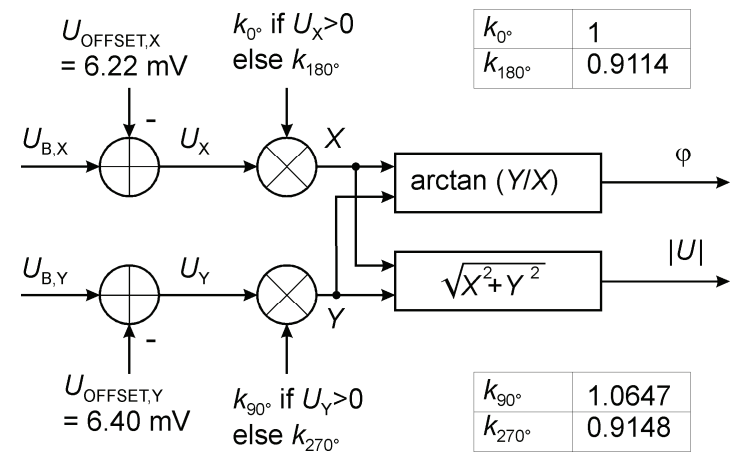

b)

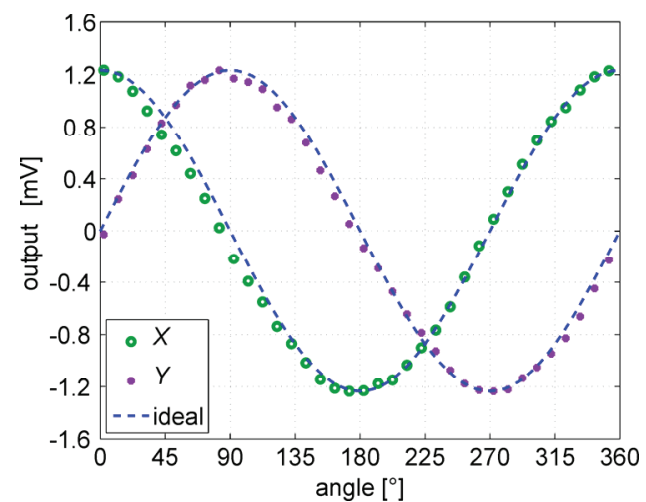

Fig. 7: a) A block diagram of the correction procedure and the subsequent data processing. b) Measured bridge voltages $U_{B, X}$ and $U_{B, Y}$ after offset and amplitude correction as a function of the flow direction $\varphi$ at $v=2 \mathrm{~m} / \mathrm{s}$ flow velocity. The dashed lines indicate ideal sine- and cosine-characteristics with $U_{X}$ at $\varphi=0^{\circ}$ as a reference amplitude.

of these compensation procedure the ideal sine- and cosine-characteristics with $U_{x}$ at $\varphi=0^{\circ}$ as a reference amplitude are also depicted.

Applying (2) and (3) to $X$ and $Y$, the flow direction $\varphi$ and the modulus of the compensated bridge voltages $|U|$ are obtained (Fig. 8). The measured flow direction plotted versus the exact values yields no perfectly straight line. To illustrate this more clearly, the angle error depending on the actual flow direction at a constant flow velocity is also plotted in Fig. 8a. The maximum angle difference over the entire range is below $8^{\circ}$, which is comparable with the results obtained from devices utilizing the pure calorimetric transduction principle $[3,8]$. The modulus of the compensated bridge voltages $|U|$ in comparison with reference value as a function of flow direction is depicted in Fig. $8 \mathrm{~b}$. The single error peak at $\varphi=45^{\circ}$ indicates the predominace of errors introduced by the sensor mounting and measurement setup. Except for the flow directions around $\varphi=45^{\circ}$, the relative error does not exceed the value of $5 \%$ over the entire angle range.

a)

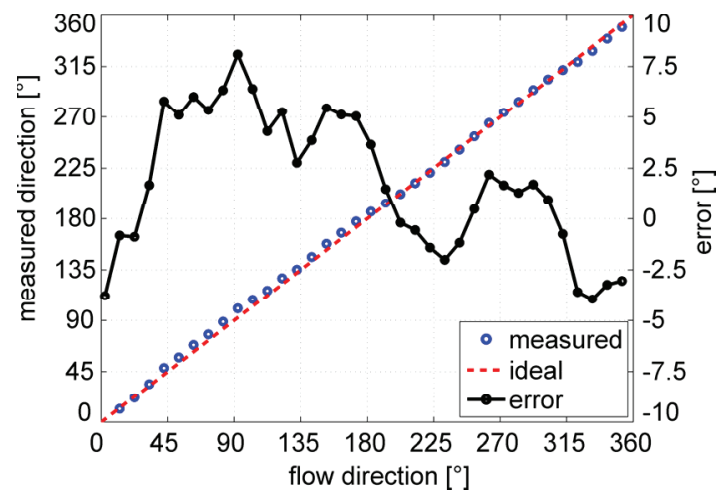

b)

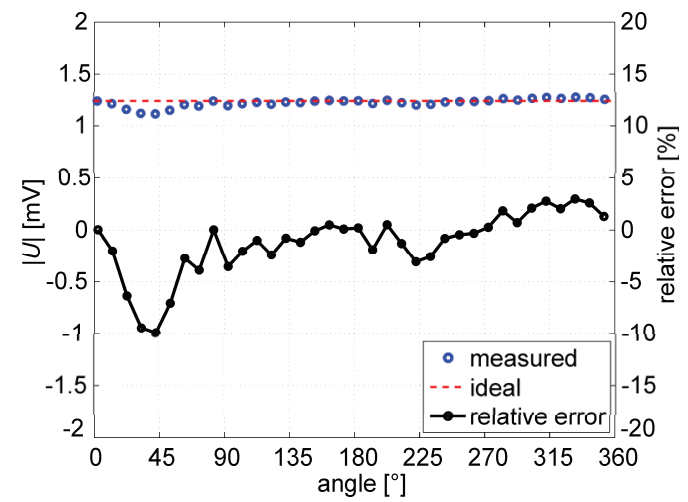

Fig. 8: a) Estimated direction versus actual flow direction at $v=2 \mathrm{~m} / \mathrm{s}$ flow velocity and the corresponding angle error. b) Measured modulus of the compensated bridge voltages $|U|$ in comparison with the reference value as a function of the flow direction at $v=2 \mathrm{~m} / \mathrm{s}$ flow velocity and the corresponding relative error.

\section{Conclusion}

We presented a micromachined flow sensor for angular-resolved flow measurements. The sensor is based on a circular Ge-thermistor array embedded in a thin membrane. Two orthogonally arranged ensambles are connected to a double Wheatstone bridge supplied with a constant current. The selfheating of the thermistors was utilized as a heat source. Due to orthogonal arrangement, the directional characteristic of the two bridge outputs can be well approximated by cosine functions exhibiting 90 degree phase shift. Therefore, the bridge voltages offer information about the $x$ - and $y$-component of the flow velocity enabling the estimation of the flow direction.

The sensor was characterized at constant flow velocity. The measured directional characteristics are subjected to an offset accompanied with a difference in the peak-to-peak values. These effects are mainly caused by the asymmetrical sensor layout and measurement setup. The corresponding systematic errors were taken into account by means of a simple correction procedure performed by the subsequent data 
processing. As a result, offset-free, approximate sinusoidal characteristics were obtained. The maximum directional error is about $8^{\circ}$ whereas the relative error of the bridge voltage modulus, which corresponds to the flow velocity magnitude, does not exceed the $10 \%$ mark. Owing to a very low supply current $(8 \mu \mathrm{A})$, the total dissipated power amounts to only $35 \mu \mathrm{W}$, corresponding to an over-temperature of the sensor membrane of less than $0.5 \mathrm{~K}$. Low power consumption is beneficial for remote sensing application whereas moderate excess temperatures can be crucial for measuring the flow of fluids that endure only slight temperature elevations (e.g., by some microfluidic application). Moreover, the effect of the natural convection causes only a negligible offset.

\section{Outlook}

Our aim was to proof the feasibility of the 2D (i.e., angular-resolved) flow measurement utilizing selfheated Ge-thermistors. This concept has been already successfully employed in 1D measurements [9]. It enables high sensitivity accompanied with extremely low power consumption. One part of the relatively high error obtained in the course of this work (10\% magnitude error and $8^{\circ}$ angle error) arises due to the thermal asymmetry induced by the inactive circular heater. Originally, the heater was planned in order to compare the results with the standard calorimetric mode. In this mode the heater is activated and the bridges are supplied with a low constant voltage. Hence, the self-heating of the thermistors can be neglected. The obtained characteristics are comparable, whereas the power consumption in the calorimetric mode is at least one order of magnitude higher due to the heater dissipation [8].

The future work shall concentrate on the new sensor design optimized only for self-heated Wheatstone bridges. The heater will then be omitted and the thermistors redesigned in order to reduce the angle error and to improve the transduction characteristics. Also the impact of the ambient temperature variation shall be considered. As both the $x$ - and $y$-component of the output signal has the same temperature dependence, the angle characteristic is not affected by temperature variations. However, the magnitude of the bridge output signal is strongly temperature dependent. Hence, if a precise measurement of the flow velocity is of the interest, the variation of the ambient temperature must be taken into account.

\section{Acknowledgements}

We gratefully acknowledge partial financial support by the Austrian Science Fund FWF (research grants L657-N16 and L234-N07).

\section{References}

[1] R. H. Ma, P. C. Chou, Y. H. Wang, T. H. Hsueh, L. M. Fu, C. Y. Lee, "A microcantilever-based gas flow sensor for flow rate and direction detection", Mycrosystem Technolgies, 15, 1201-1205 (2009).

[2] K. Seunghyun, N. Teckjin, and P. Sekwang, "Measurement of flow direction and velocity using a micromachined flow sensor", Sensors and Actuators, A 114, 312-318 (2004).

[3] B.W. van Oudheusden, and J. H. Huijsing, "An electronic wind meter based on a silicon flow sensor", Sensors and Actuators, A 21-23, 420-424 (1990).

[4] F. Keplinger, J. Kuntner, A. Jachimowicz, F. Kohl, and B. Jakoby, "Highly sensitive sensor for flow velocity and flow direction measurement”, Proceedings 5th IEEE Conference on Sensors, Daegu, Korea, 1436-1439 (2006).

[5] A. Cubukcu, E. Zernickel, U. Buerklin, G. Urban, "A 2D thermal flow sensor with sub-mW power consumption", Sensors and Actuators, A 163, 449-456 (2010).

[6] F. Kohl, R. Fasching, F. Keplinger, R. Chabicovsky, A. Jachimowicz, G. Urban, "Development of miniaturized semiconductor flow sensors", Measurement, 33, 109-119 (2003).

[7] R. Beigelbeck, F. Kohl, J. Kuntner, B. Jakoby, "Heat transfer analysis of micromachined thermal conductivity sensors", Proceedings ETFA 2005, Catania, Italy, 977-983 (2005).

[8] S. Cerimovic, A. Talic, F. Kohl, J. Schalko, F. Keplinger, "Micromachined Wind Sensors Based on a Circular Thermistor Array in a Double Bridge Configuration”, Proceedings ICST2010, Lecce, Italy, 495-500 (2010).

[9] A. Talic, S. Cerimovic, R. Beigelbeck, F. Kohl, A. Jachimowicz, F. Keplinger, "Novel Thermal Flow Sensors Based on a Wheatstone Bridge Read-out", proceedings of EUROSENSORS 2009, Lausanne, Switzerland, 136-139 (2009). 